\title{
Numerical Modeling of Flow in Fishways Embedded with Circular/Rectangular Obstructions
}

\author{
Subas Chhatkuli ${ }^{1}$ and Masayuki Fujihara ${ }^{2}$
}

\begin{abstract}
Two dimensional shallow water equations are solved by applying a Godunov-type numerical method to analyze the flows in fishways with obstructions of circular and rectangular shapes of equal area, embedded with regular and staggered patterns. Results show that obstructions embedded with a regular pattern creates shorter fish pathways and larger low velocity regions although flow is accompanied with a little higher velocity at the center of gaps. Water depth and its distribution pattern in a pool are almost the same when the obstructions with circular/rectangular shapes are embedded with a regular pattern. However, obstructions embedded with a staggered pattern create alternate shallower and deeper pools in fishways.
\end{abstract}

Keywords: Flow simulation; Shallow water; Finite volume method; Fish; Computational fluid dynamics

\section{Introduction}

Nature-like fishways bypass hydraulic structures such as dams and are patterned after natural river channels. These fishways essentially consist of a sloping channel embedded with a set of obstructions. Wake regions in the channel provide shelter from swift currents, which fish use to rest during upstream migration.

Recently, nature-like fishways are becoming popular as they are in better harmony with natural river systems and provide easier passage to fish which do not prefer to jump. Moreover, if the construction materials are locally available, nature-like fishways will be more economical to construct than the conventional fishways (Harris et al. 1999).

The layout and the shape of obstructions control water levels and the distribution of velocity which are critical to fish habitat. Fishway designers must determine the layout, shape, spacing and orientation of obstructions based on site-specific constraints and there is presently a little research to support design (Acharya et al. 2000; Kells et al. 2000).

When a bluff object or a solid block is placed along the flow direction, the object blocks a significant part of the flow area. The flow decelerates as it approaches the block and then it separates and spreads around the bluff object. Near the free surface in front of the bluff object, the upward flow forms a circulation causing the depth to increase in front of the object. Behind the bluff object the pressure decreases due to strong circulation and the water level decreases. The water depth increases gradually away from the bluff object until it reaches the flow depth not influenced by the presence of the bluff object (Salaheldin et al. 2004).

When these bluff objects are installed in a series at an interval, they act as a nature-like fishway. The flow proper-

\footnotetext{
${ }^{1}$ Researcher, Faculty of Engineering, University of Tokyo, 7-3-1 Hongo, Bunkyo-ku, Tokyo 113-8656, Japan

${ }^{2}$ Professor, Faculty of Agriculture, Ehime University, 3-5-7 Tarumi, Matsuyama, Ehime, 790-8566 Japan (Corresponding Author) fujihara@agr.ehime-u.ac.jp
}

ties depend on the shape, area, and the pattern of embedment of these obstructions. Therefore, a numerical model which can simulate the flow around these series of block objects will be an economical tool in designing these kinds of fishways.

Nature-like fishways are designed considering the obstructions would not submerge for the majority of the discharge condition so that the leeward side of the obstructions would provide a refuge to ascending fish. Nature-like fishways, under non-submergence conditions, behave like a natural version of vertical slot fishway. Researches on vertical slot fishways have shown that when the slope of the fishway is $5 \%$, the flow in a pool is two-dimensional (2D) and the vertical component of the velocity is generally negligible (Wu et al. 1999; Puertas et al. 2004).

In nature-like fishways, slope of 5\% appears to be the most common (Kells et al. 2000). In this work, the study is performed on a nature-like fishways with slope $5 \%$. Hence flows in such kind of fishways can be considered 2D and the depth averaged model could be used confidently in such cases.

However, in vertical slot fishways, when the slopes are $10 \%$ or $20 \%$, flow is three-dimensional (3D). Hence it can be assumed that in nature-like fishways with slope $10 \%$ or $20 \%$, flow would be $3 \mathrm{D}$. In such cases the use of depth averaged model could lead to erroneous results due to the 3D nature of the flow. Therefore, further investigation is needed to find out the limitations of the model for higher slopes.

The purpose of this paper is to compare two generic design approaches that involve regular and staggered patterns of obstructions, and to examine the advantages and disadvantages of rectangular vs. circular obstructions. Furthermore, this paper highlights the utility of modern computational methods in the context of hydraulic design. A Godunov-type numerical model of depth averaged two dimensional Shallow Water Equations (SWEs) has been applied to get the insight of this poorly understood yet very inter- 
esting flow phenomenon, through four test cases.

\section{Governing equations}

SWEs describe flow in shallow water bodies where the vertical acceleration within the fluid can be neglected and then the pressure becomes hydrostatic. Hence the continuity of mass and momentum equations can be integrated over the depth and solved numerically to give the depth averaged velocity fields.

Depth averaged conservation of mass and momentum equations used are as follows (Rogers et al. 2001),

$$
\frac{\partial \mathbf{Q}}{\partial t}+\nabla \cdot \mathbf{F}=\mathbf{H}
$$

in which

$$
\begin{aligned}
& \mathbf{Q}=\left[\begin{array}{l}
\zeta \\
u h \\
v h
\end{array}\right], \\
& \mathbf{F}=\left[\begin{array}{ll}
u h & v h \\
u^{2} h+\frac{1}{2} g\left(\zeta^{2}+2 \zeta h_{s}\right)-v h \frac{\partial u}{\partial x} & u v h-v h \frac{\partial u}{\partial y} \\
u v h-v h \frac{\partial v}{\partial x} & v^{2} h+\frac{1}{2} g\left(\zeta^{2}+2 \zeta h_{s}\right)-v h \frac{\partial v}{\partial y}
\end{array}\right]
\end{aligned}
$$$$
\text { and } \mathbf{H}=\left[\begin{array}{l}
0 \\
-\frac{\tau_{b x}}{\rho}-g \zeta S_{o x} \\
-\frac{\tau_{b y}}{\rho}-g \zeta S_{o y}
\end{array}\right] \text {, }
$$

where $\zeta$ is the free surface elevation above the still water level $h_{s}, h\left(=\zeta+h_{s}\right)$ the total water depth, $u$ and $v$ the depth-averaged velocities in the $x$ and $y$ directions respectively, $u_{x}, u_{y}$ and $v_{x}, v_{y}$ the derivatives of the depth averaged velocity components in the $x$ and $y$ directions respectively, $g$ the acceleration due to gravity, $\rho$ the water density, $\tau_{b x}$ and $\tau_{b y}$ the bed friction stresses in the $x$ and $y$ directions respectively, $v$ the kinematic eddy viscosity coefficient and $S_{o x}$ and $S_{o v}$ the bed slopes in the $x$ and $y$ directions respectively.

The bed shear stress terms are evaluated as

$$
\begin{aligned}
\tau_{b x} & =\frac{\rho g m^{2} u \sqrt{u^{2}+v^{2}}}{h^{1 / 3}} \\
\tau_{b y} & =\frac{\rho g m^{2} v \sqrt{u^{2}+v^{2}}}{h^{1 / 3}}
\end{aligned}
$$

\section{Numerical modeling}

Integrating Eq. (1) over a control volume $(\Omega)$ and by applying Gauss' theorem we get

$$
\frac{\partial}{\partial t} \int_{\Omega} \mathbf{Q} d \Omega+\oint_{\Psi} \mathbf{F} \cdot \mathbf{n} d \Psi=\int_{\Omega} \mathbf{H} d \Omega
$$

where $\Psi$ the boundary of $\Omega$ and $\mathbf{n}$ the unit normal vector through $\Psi$.

The flux in Eq. (4) can be written in terms of inviscid and viscid form

$$
\mathbf{F} \cdot \mathbf{n}=\mathbf{f}^{I}-u \mathbf{f}^{V}
$$

in which

$$
\mathbf{f}^{I}=\left[\begin{array}{l}
u h n_{x}+v h n_{y} \\
\left(u^{2} h+g\left[\zeta^{2}+2 \zeta h_{s}\right] / 2\right) n_{x}+u v h n_{y} \\
u v h n_{x}+\left(v^{2} h+g\left[\zeta^{2}+2 \zeta h_{s}\right] / 2\right) n_{y}
\end{array}\right]
$$

and $\mathbf{f}^{V}=\left[\begin{array}{l}0 \\ (h \partial u / \partial x) n_{x}+(h \partial u / \partial y) n_{y} \\ (h \partial v / \partial x) n_{x}+(h \partial v / \partial y) n_{y}\end{array}\right]$,

where $n_{x}$ and $n_{y}$ are Cartesian components of $\mathbf{n}$.

The SWEs are discretised on q-tree girds (Rogers et al. 2001) with conserved variables ( $\zeta, u h$ and $v h$ ) stored at the cell center of each computational cell. The inviscid fluxes are evaluated by adopting Roe's approximate Riemann solver (Roe 1981) at each cell edge. The inter cell inviscid flux $\mathbf{f}_{i, j}^{I}$ is evaluated as

$$
\mathbf{f}_{i, j}^{I}=1 / 2\left[\mathbf{f}^{I}\left(\mathbf{Q}_{i, j}^{+}\right)+\mathbf{f}^{I}\left(\mathbf{Q}_{i, j}^{-}\right)-|\mathbf{A}|\left(\mathbf{Q}_{i, j}^{+}-\mathbf{Q}_{i, j}^{-}\right)\right]
$$

in which

$$
|\mathbf{A}|=\mathbf{R}|\mathbf{\Lambda}| \mathbf{L}
$$

where $\mathbf{Q}_{i, j}^{+}$and $\mathbf{Q}_{i, j}^{-}$are reconstructed right and left Riemann states respectively at the cell interface located between adjacent cells $i$ and $j$ and $\mathbf{A}$ the flux Jacobian using $\mathbf{R}$ and $\mathbf{L}$, the right and left eigenvector matrices of $\mathbf{A}$ respectively. Right and left eigenvector matrices are

$$
\mathbf{R}=\left(\begin{array}{ccc}
0 & 1 & 1 \\
n_{y} & u-c n_{x} / n & u+c n_{x} / n \\
-n_{x} & v-c n_{y} / n & v+c n_{y} / n
\end{array}\right)
$$

where $m$ is the Manning's coefficient. 


$$
\mathbf{L}=\left(\begin{array}{ccc}
-\frac{\left(u n_{y}-v n_{x}\right)}{n^{2}} & \frac{n_{y}}{n^{2}} & \frac{n_{x}}{n^{2}} \\
\frac{\left(u n_{x}+v n_{y}\right)}{2 c n}+\frac{1}{2} & \frac{-n_{x}}{2 c n} & \frac{-n_{y}}{2 c n} \\
-\frac{\left(u n_{x}+v n_{y}\right)}{2 c n}+\frac{1}{2} & \frac{n_{x}}{2 c n} & \frac{n_{y}}{2 c n}
\end{array}\right) .
$$

The symbol $|\Lambda|$ in Eq. (10) denotes a diagonal matrix of the absolute values of the eigenvalues of $\mathbf{A}$, expressed as

$$
|\boldsymbol{\Lambda}|=\left(\begin{array}{ccc}
\left|u n_{x}+v n_{y}\right| & 0 & 0 \\
0 & \left|u n_{x}+v n_{y}-c n\right| & 0 \\
0 & 0 & \left|u n_{x}+v n_{y}+c n\right|
\end{array}\right)
$$

where $c$ is the wave celerity and $n=\sqrt{n_{x}^{2}+n_{y}^{2}}$.

Variables $u, v, c$ in Eqs. (8) - (10) are given by Roe's average state defined as

$$
\begin{aligned}
& u=\frac{u^{+} \sqrt{h^{+}}+u^{-} \sqrt{h^{-}}}{\sqrt{h^{+}}+\sqrt{h^{-}}}, v=\frac{v^{+} \sqrt{h^{+}}+v^{-} \sqrt{h^{-}}}{\sqrt{h^{+}}+\sqrt{h^{-}}}, \\
& \text {and } c=\sqrt{\frac{g\left(h^{+}+h^{-}\right)}{2}}
\end{aligned}
$$

where the superscripts + and - denote the right and left Riemann states on either side of a cell interface respectively. The viscous fluxes are evaluated by using central difference method and fourth order Runge-Kutta scheme is used for the temporal integration.

Kinematic eddy viscosity is evaluated by using the Smagorinsky model as expressed in Eq. (11)

$$
v=\left(C_{s} \Delta\right)^{2}\left[2\left(\frac{\partial u}{\partial x}\right)^{2}+\left(\frac{\partial u}{\partial y}+\frac{\partial v}{\partial x}\right)^{2}+2\left(\frac{\partial v}{\partial y}\right)^{2}\right]^{1 / 2}
$$

in which

$$
\Delta=(\Delta x \cdot \Delta y)^{1 / 2}
$$

where $\Delta x, \Delta y$ are the computational cell lengths in the $x$ and $y$ directions respectively and $C_{s}$ the Smagorinsky constant. The Smagorinsky constant can be chosen in a range from 0.10 to 0.30 and it is required to have a sensible value to avoid excessive damping of resolved structures (Tutar and Holdo, 2001). Though the Smagorinsky model was originally developed for a three dimensional simulation, it is employed here due to its less complexity. Moreover, this model reduces the computational load compared to other turbulence closure models.

The minmod limiter is selected as a slope limiter in a computational cell where the values of variables are linearly distributed so that the numerical scheme employed be- comes a second-order approximation. Furthermore, numerical experiments show that the use of minmod limiter can produce more stable results.

\section{Boundary conditions}

At solid boundaries the no slip condition is applied. In other words, velocity normal to solid boundary is set zero to imply no flux through boundaries and the tangential component of velocity at the solid boundary is also set zero.

Riemann invariants, specified according to the local Froude number, are used to implement open boundary condition as follows:

(i) subcritical flow (when water depth $h_{B}$ is imposed),

$$
\tilde{u}_{B}=\tilde{u}_{I}+2 \sqrt{g}\left(\sqrt{h_{I}}-\sqrt{h_{B}}\right), \quad \tilde{v}_{B}=\tilde{v}_{I}
$$

(ii) subcritical flow (when the velocity $\widetilde{u}_{B}$ is imposed),

$$
h_{B}=\left(\frac{1}{2 \sqrt{g}}\left(\widetilde{u}_{I}-\widetilde{u}_{B}\right)+\sqrt{h_{I}}\right)^{2}, \widetilde{v}_{B}=\widetilde{v}_{I}
$$

(iii) supercritical inflow (the variables $\left(h_{B}, \widetilde{u}_{B}, \widetilde{v}_{B}\right)$ are given), and

(iv) supercritical outflow

$$
h_{B}=h_{I}, \tilde{u}_{B}=\tilde{u}_{I}, \widetilde{v}_{B}=\widetilde{v}_{I} .
$$

In the above equations, subscript $B$ denotes the boundary value, subscript $I$ denotes the inner Riemann state value at the boundary, $\widetilde{u}$ and $\widetilde{v}$ are the depth-averaged velocity components normal and tangential to the boundary respectively.

\section{Model validation}

The model validation is performed to check the ability of the model to simulate flow around obstructions and in the wake regions accurately. For the validation purpose, vortex shedding from circular cylinders (Case 1) and two test cases (Case 2 and Case 3) for which experimental data have been reported in literatures are simulated.

In case 1, the numerical scheme is used to simulate the vortex shedding past a circular cylinder of $74 \mathrm{~mm}$ diameter $(D)$ in a $5 \mathrm{~m}$ long and $0.5 \mathrm{~m}$ wide flume. The velocity in the far field of the diameter is $U=0.22 \mathrm{~m} / \mathrm{s}$, the corresponding Reynolds number is $R e=16,428$ and the vortex shedding frequency is $f_{v}=0.6667 \mathrm{~Hz}$. The frequency is calculated from the fluctuation of the transversal velocity component. Figure 1 shows time dependent velocity vectors at every quarter cycle. At this condition, flow is sub-critical throughout. Schlichting (1979) reported that at the higher Reynolds number $\left(150-300<R e<1 \times 10^{5}-1.3 \times 10^{5}\right)$, in the sub-critical range, Strouhal number $\left(S t=f_{v} D / U\right)$ remains approximately constant at $S t=0.21$. The Strouhal number obtained by the simulation is 0.22 , which corresponds reasonably close to the experimental results. 


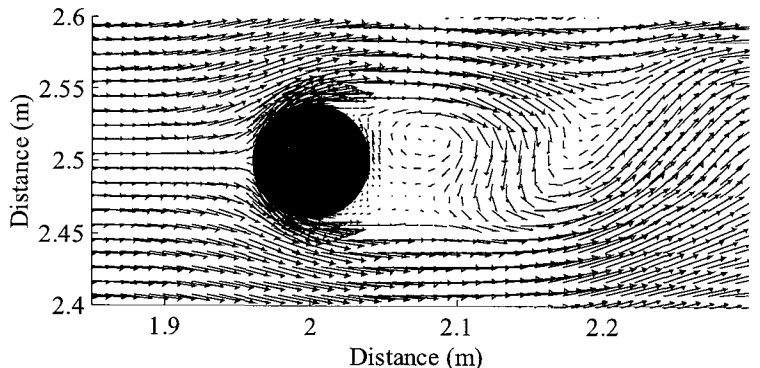

$\frac{\mathrm{T}}{4}$

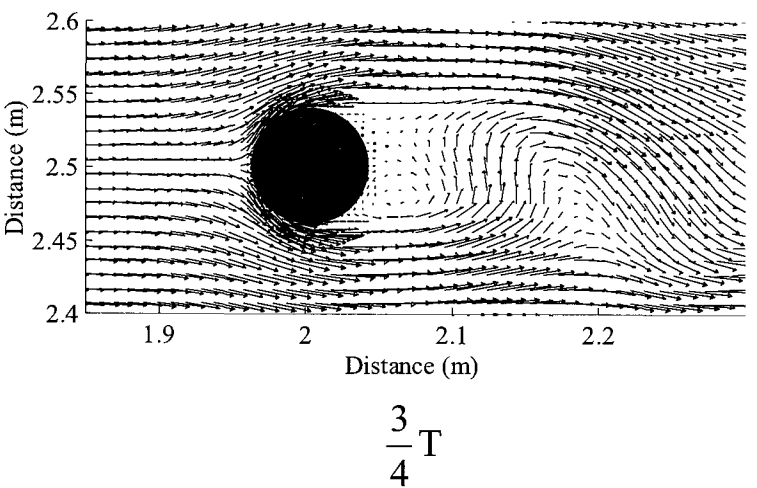

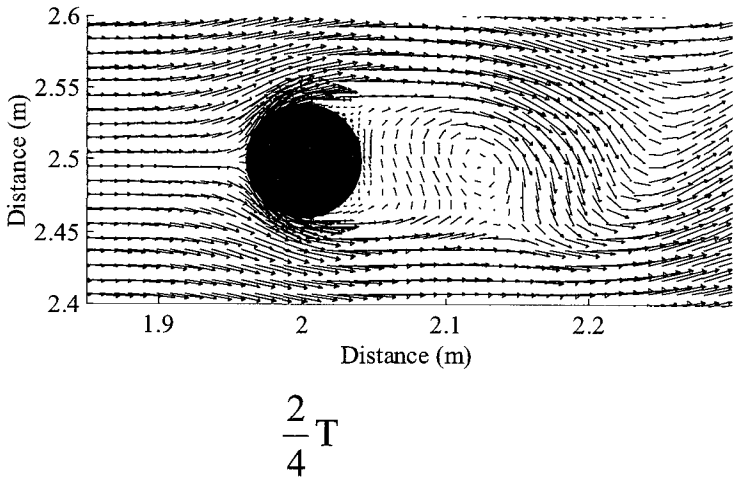

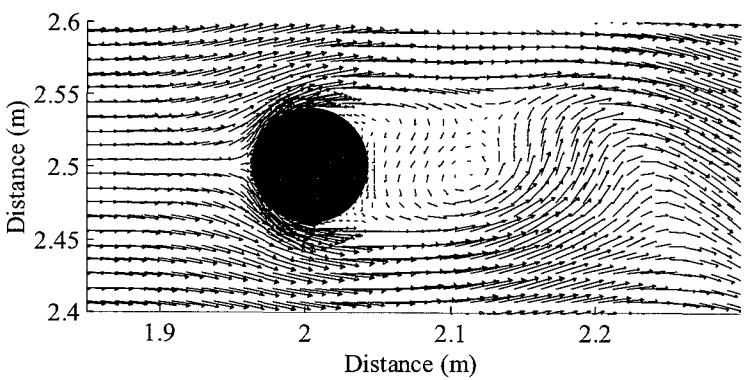

$\mathrm{T}$

Figure 1 : Time dependent velocity vectors showing the vortex shedding from a cylinder

Case 2 involves modeling of sub-critical flow around a cylinder of $74 \mathrm{~mm}$ diameter in a $5 \mathrm{~m}$ long and $0.5 \mathrm{~m}$ wide flume reported in Acharya et al. (2000). Normalized velocities along the centre of the cylinder and along the transverse section are compared with the experimental data.

Inflow velocity is set $0.303 \mathrm{~m} / \mathrm{s}$ and inflow depth is set $42.92 \mathrm{~mm}$ as a boundary condition. Hence, the Froude number results in 0.468 , which corresponds to the physical experimental case.

Figure 2 shows the normalized velocity for the cylindrical column. In Figure 2, Vo corresponds to the inflow velocity, $V$ the point velocity at the measured section, $X$ the longitudinal distance from the center of the cylinder and $D$ the diameter of the cylinder. Along the longitudinal section, $90 \%$ velocity recovery is obtained at the distance of $8 D$ from the center of the cylinder, in the both experimental and computational observations. Along the transverse sections, gradual decrement and increment in velocities were observed along $X=0$ and $X=6 D$ (Figure 3) respectively, from the centre of the cylinder. In Fig. 3, $Y$ is the transverse distance from the center of the cylinder. In all the three cases, computational results are in a good agreement with the experimental results.

Case 3 involves modeling of flow behind side-by-side cylinders at small gap ratios. The time averaged velocity vectors obtained by the simulation are compared with the data reported in the literature (Akili et al. 2004). The aim of this experiment is to test the effect of the gap width of the cylinder on the wake region, against the prior works available in the literature. When the ratio of distance between the cylinders $(L)$ and the cylinder diameter $(D)$ is upto 1.5 , the previous works showed that, the two side-by-side cylinders

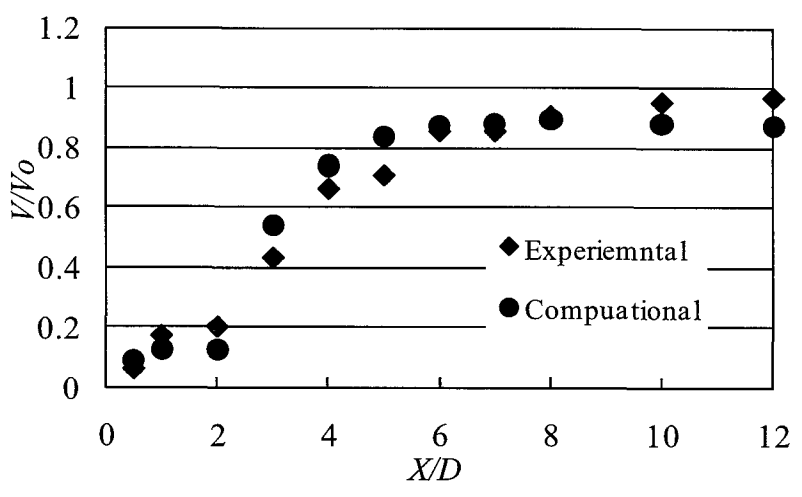

Figure 2 : Normalized velocity for cylindrical column, longitudinally

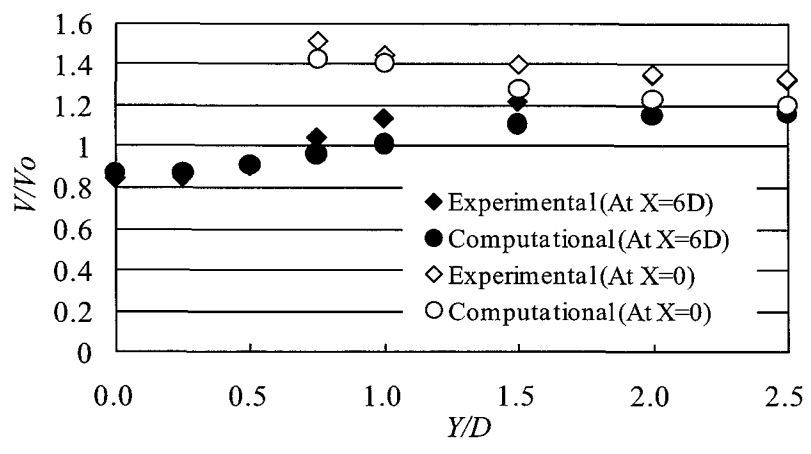

Figure 3 : Normalized velocity for cylindrical column, transversely along $X=0$ and $X=6 D$ 
works collectively. However, when the $L / D$ ratio is 2.5 or more than that the two cylinders act independently.

Numerical simulation is carried out by keeping the flow depth, at the boundaries, $h=25 \mathrm{~mm}$, and the diameter of the cylinder, $D=30 \mathrm{~mm}$, the same as in the experiments conducted by Akilli et al. (2004). The width of the flume is also set $1 \mathrm{~m}$ as in the experiment.

Figure 4 shows the time averaged velocity vector fields, obtained by the simulation, for the cases $L / D=1.25,1.5$ and 2.5. Simulation results showed the asymmetrical flow pattern for the lower gap ratio $(L / D=1.25$ and 1.5) and the deflection of the jet flow was towards the narrow wake region. Akili et al. (2004) observed that the flow deflection was not always in the upper side of the cylinder though this nature was completely arbitrary. This intrinsic property of fluid flow was encountered by Bearman and Waddock (1973), Kim and Durbin (1988) and Sumner et al. (1999) as well.

As also seen in Figure 4, when $L / D=2.5$, the simulation result shows that the side-by-side circular cylinders behave more as an independent isolated bluff objects supporting the finding of Akili et al. (2004). Therefore, the results for Case 3 show this numerical model can reproduce the effect of gap width of the cylinder on the wake region.

These results are in good agreement with the experimental results. Hence, the model can be used confidently for the numerical experimental purpose. However, if water depth is too shallow, at some point vortex shedding will probably stop due to the very high bed friction stress.

\section{Experimental cases}

The aim of the numerical experiments is to analyse the change in flow properties due to the shape of obstruction and the pattern of embedment. The numerical analysis of flows is performed on ramp fishways, embedded with circular/rectangular obstructions, having length and width 25 $\mathrm{m}$ and $2 \mathrm{~m}$ respectively. The slope of the fishway was set $1: 20$ from $1 \mathrm{~m}$ to $21 \mathrm{~m}$. The first $1 \mathrm{~m}$ and the last $4 \mathrm{~m}$ are horizontal. The schematic diagram is depicted in Figure 5.

Circular and rectangular obstructions are embedded in the fishways with regular and staggered patterns. The Radius of the circular obstruction is set as $0.2 \mathrm{~m}$ and the area of a rectangular obstruction $(0.4 \mathrm{~m} \times 0.3 \mathrm{~m})$ is kept equal to the area of a circular one. For the regular cases, obstructions are kept at every $1 \mathrm{~m}$ interval center to center from 1 $\mathrm{m}$ to $21 \mathrm{~m}$, longitudinally, and three obstructions were kept, transversely, such that the gaps between the edge of obstructions and between the guide wall and obstruction are $0.2 \mathrm{~m}$. For the staggered cases, longitudinal set up of the obstructions are the same as in the regular case. However, along the transverse section, four obstructions are embedded such that the distance between the obstruction centers is set $0.67 \mathrm{~m}$. Hence, net gap width at any cross sections, along the center of the obstruction, is then $0.80 \mathrm{~m}$ for all the four test cases.

Obstructions set in a transverse sections act like weirs in a pool and weir type fishway and at the same time gaps between the obstructions perform the function of slots in a vertical slot fishway. The low velocity regions hence oc-
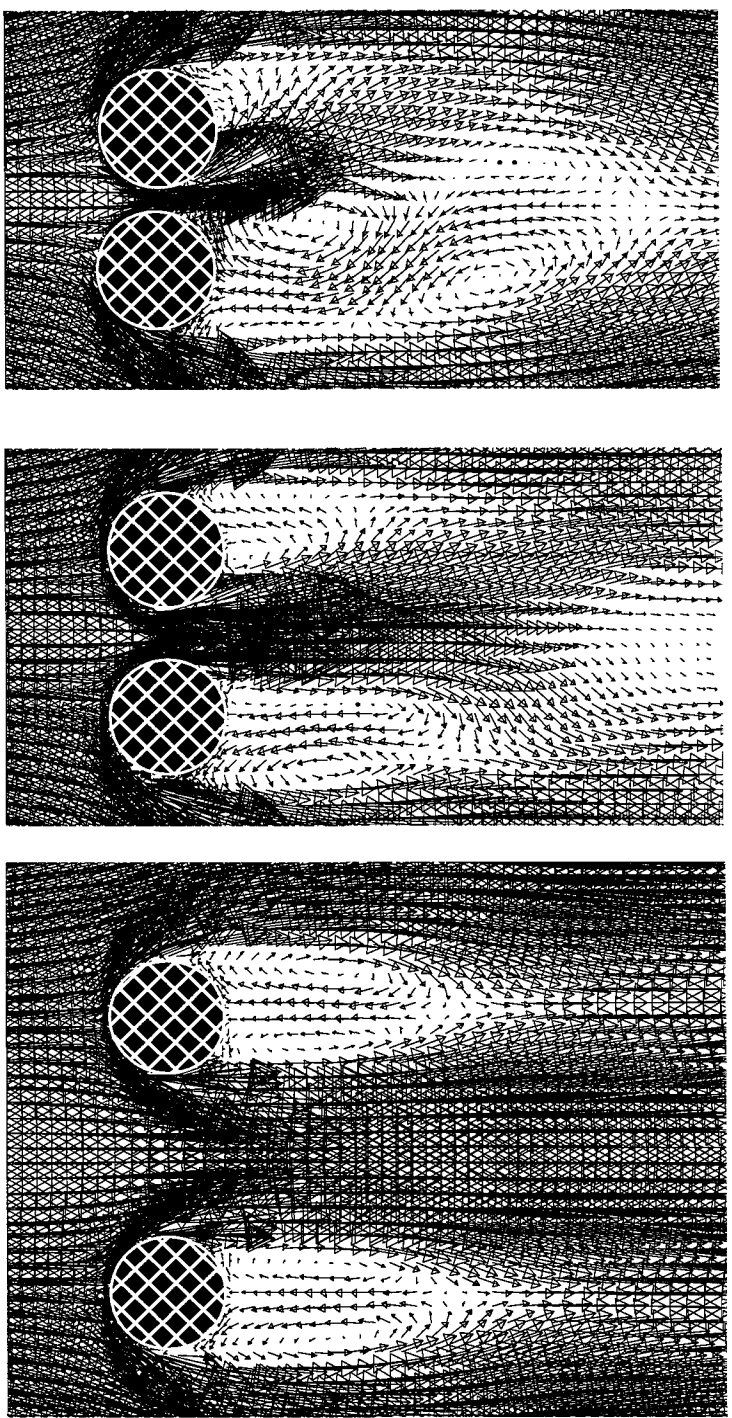

Figure 4 : Averaged velocity vectors with $L / D=$ 1.25(top), 1.5(middle), and 2.5(bottom)

curred, behind the obstructions, in the longitudinal section provide refuge to ascending fish.

In nature-like fishways, the minimum flow depth and the maximum average water velocity that fish could overcome depend on fish size species. Hence, the prior knowledge of water depth at upstream and downstream of fishways would help the designer to create a favorable flow depth in a fishway. Therefore in this study, water depths at upstream and downstream are selected as boundary conditions rather than in a conventional way of specifying the discharge at an upstream boundary and water depth at the downstream boundary. Water depths at upstream and downstream are set 0.2 $\mathrm{m}$ as a boundary condition. Height of an obstruction is kept always more than water depth to avoid the submergence case.

Bottom of a nature-like fishway is constructed by embedding small pebbles or stones so that the other invertebrates, like shrimps, may also use the fishway, to travel upstream, by clinging on the rough bottom surface of the fishway. Rice et al. (1998) recommended a predictive equa- 


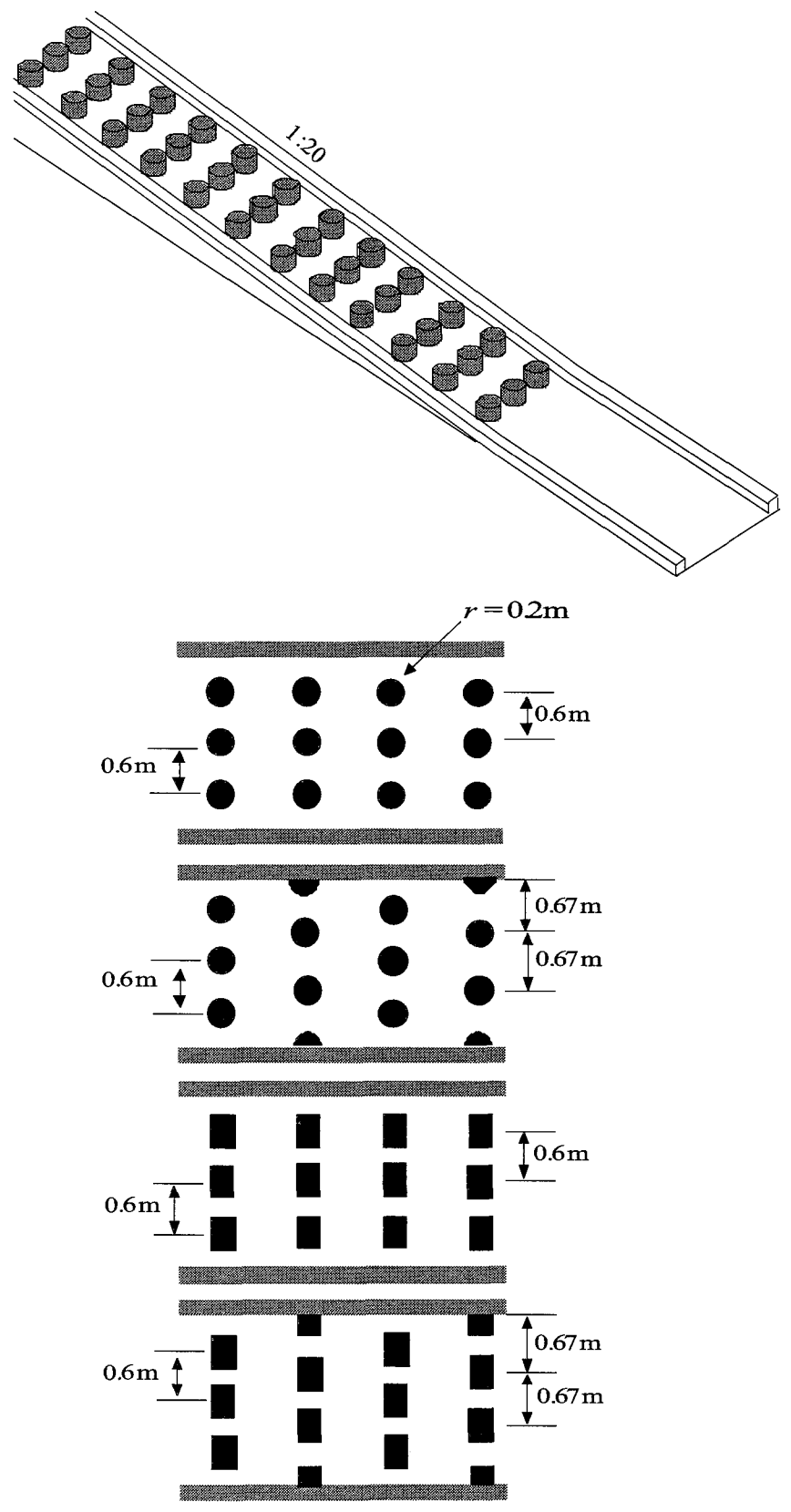

Figure 5 : Schematic isometric and plan view of fishways embedded with circular/rectangular obstructions

tion for hydraulic resistance in terms of Manning's coefficient $m$ and slope $S_{0}$, as

$$
m=0.029\left(D_{50} S_{0}\right)^{0.147}, \quad 0.028 \leq S_{0} \leq 0.33
$$

where $D_{50}$ is the stone size for which $50 \%$ is finer (mm).

The bottom roughness in the four test cases was considered rough and embedded with small pebbles of diameter $30 \mathrm{~mm}$. Hence, using Eq. (15), a Manning's coefficient of $0.03 \mathrm{~m}^{-1 / 3} \mathrm{~s}$ is assumed.

As a preliminary study, grid convergence tests are conducted using three different maximum grid sizes in a pool. Those sizes are $19.53 \mathrm{~cm} \times 19.53 \mathrm{~cm}$ (level 7), $9.76 \mathrm{~cm} \times$ $9.76 \mathrm{~cm}$ (level 8 ) and $4.88 \mathrm{~cm} \times 4.88 \mathrm{~cm}$ (level 9). In all the cases, minimum size of grid is $2.44 \mathrm{~cm} \times 2.44 \mathrm{~cm}$, which is

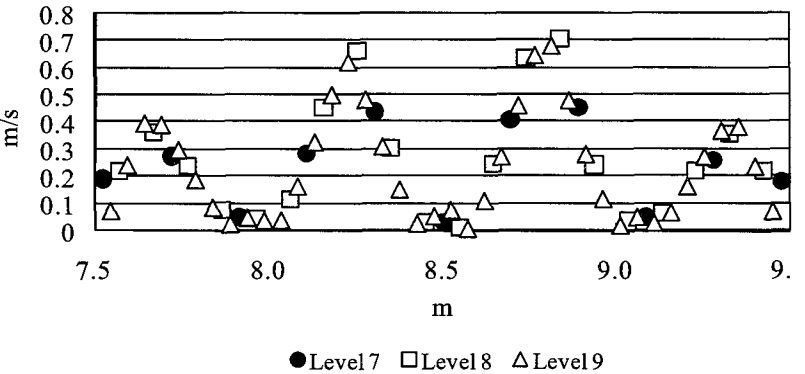

Figure 6 : Velocity along the centre of a pool (transversely) for the various grid sub-division level

allocated along the circumference of obstructions. Figure 6 shows the velocity along the transverse direction, between the guide walls of a fishway, at the centre of the pool using different grid sizes. Grid convergence tests (Figure 6) shows that the grid size level 7 would be too coarse. The resultant velocity distributions obtained by using grid sizes level 8 and level 9 are almost identical. Hence, grid size level 8 is used in this study.

\section{Results and discussion}

\subsection{Discharge}

Since the boundary depths are constant, difference in discharges satisfying the boundary conditions would show the change in overall resistance to flow in the fishways. The total discharges of $0.072 \mathrm{~m}^{3} / \mathrm{s}$ and $0.082 \mathrm{~m}^{3} / \mathrm{s}$ are required to attain the boundary conditions, for the fishways with rectangular and circular obstructions, respectively. This implies that the resistance to flow is higher in a fishway with rectangular obstructions than in fishway with circular obstructions even though the area of obstruction is the same. However, in the case of fishways with obstructions embedded with a regular and staggered pattern, almost the same discharge is required to satisfy the boundary conditions.

\subsection{Velocity}

Figures 7 and 8 show the time averaged velocity vectors in the four fishways with circular and rectangular obstructions, of equal area, embedded with regular and staggered patterns. Since the water depth and velocity distributions are identical in all the pools except the first and the last one, the results obtained in the areas between the lengths $2.5 \mathrm{~m}$ to 5.5 $\mathrm{m}$ are taken as representative. From the figures, small vortexes are seen behind the obstructions, in the wake regions, and a maximum velocity at the center of gaps between the obstructions. Flow velocities, along the upstream perimeter of obstructions, are higher in the fishways with obstructions embedded with a staggered pattern than those with a regular pattern.

Equi-velocity lines in Figures 9 and 10 show the trend of velocity distribution in the fishways. The trend roughly followed the similar patterns in the fishways with circular and rectangular obstructions. The maximum velocity, which is over $1 \mathrm{~m} / \mathrm{s}$, is found at the center of the gap, between the obstructions. Whereas, due to the solid roughness, 

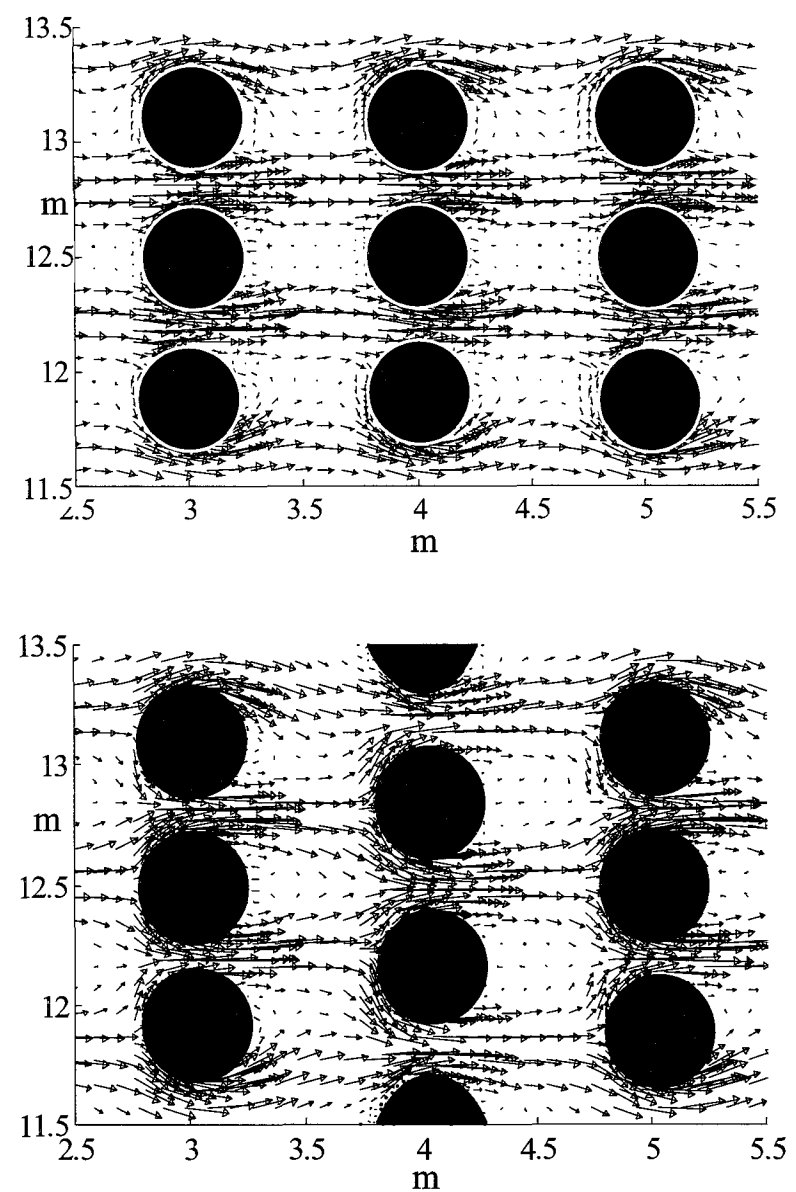

Figure 7 : Velocity vectors in fishways embedded with circular obstructions

the closer to the gap edge the lower the velocity is found. As the flow passed through the gap, the flow becomes calmer. In all the cases, low velocity regions are seen behind the obstructions.

Bar charts in Figures 11 and 12 show a detail picture of the velocity $(\mathrm{m} / \mathrm{s})$ distribution in the flow domain of the fishways. The low velocity regions, where velocity was less or equal to $0.1 \mathrm{~m} / \mathrm{s}$, are larger in the case of fishways with rectangular obstructions. Likewise, the maximum velocity was reduced when the obstruction shape was changed to rectangular. Moreover, the low velocity region is larger when the obstructions are embedded with a regular pattern. In the fishways, the staggered pattern causes the meandering of the flow, reducing the low velocity region. Nevertheless, the same meandering flow decreases the maximum velocity to some extent.

\subsection{Water depth and level}

Water depth contours, in fishways, are shown in Figures 13 and 14. The distributions of water depth patterns, in fishways with circular and rectangular obstructions, are very similar when the upstream boundary depths are equal.

Upstream perimeters of the obstructions are accompanied with the deeper water regions. And, as the flow enters the gap, water depth starts to decrease and attains a minimum value at the downstream half of the gap, between the ob-
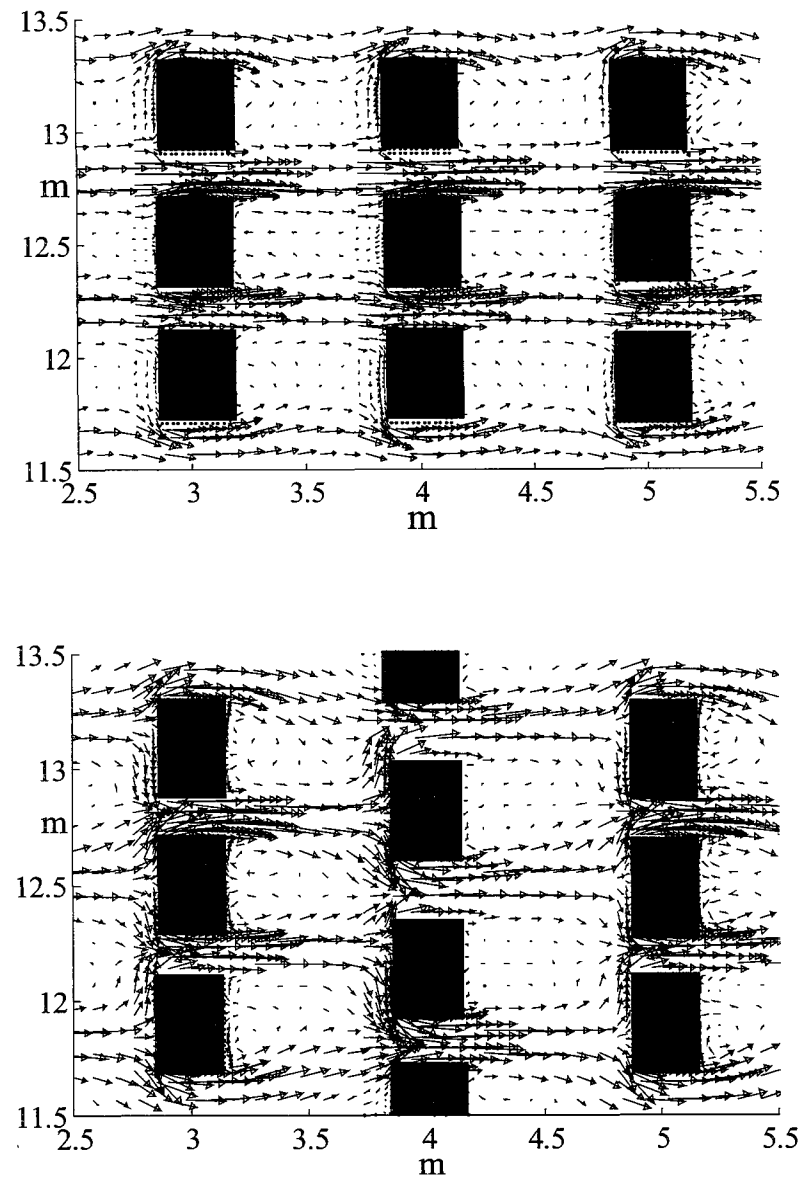

Figure 8 : Velocity vectors in fishways embedded with rectangular obstructions

structions. The water depth starts to recover after it exits from the gap.

The effect of the gap opening is clearly seen in the staggered patterned fishways. The gap width, between the obstructions, in the case of three obstructions in a line is $0.2 \mathrm{~m}$ and, in the case of four obstructions, $0.267 \mathrm{~m}$. However, the total gap width in both the cases is equal to $0.8 \mathrm{~m}$. The results showed that average water depth in a pool in front of three gaps with total opening $0.8 \mathrm{~m}$ is shallower than in a pool in front of four gaps with total opening of $0.8 \mathrm{~m}$. This implies each opening acted independently not as a series of openings collectively.

Free surface configuration in a part of the fishway with circular obstructions embedded with a regular pattern is shown in Figure 15. The values in the vertical axis denote the distance below a reference level. Figure 15 shows that the free surface is almost horizontal in the pool and the drop in water level occurs only at the gap between the obstructions. The average gradient of the water surface through fishways is nearly equal to the gradient of the bottom slope. This is true in the other three cases as well.

\subsection{Discussion}

The method used to analyze the flow properties, in nature-like fishways, is based on a numerical modeling of 

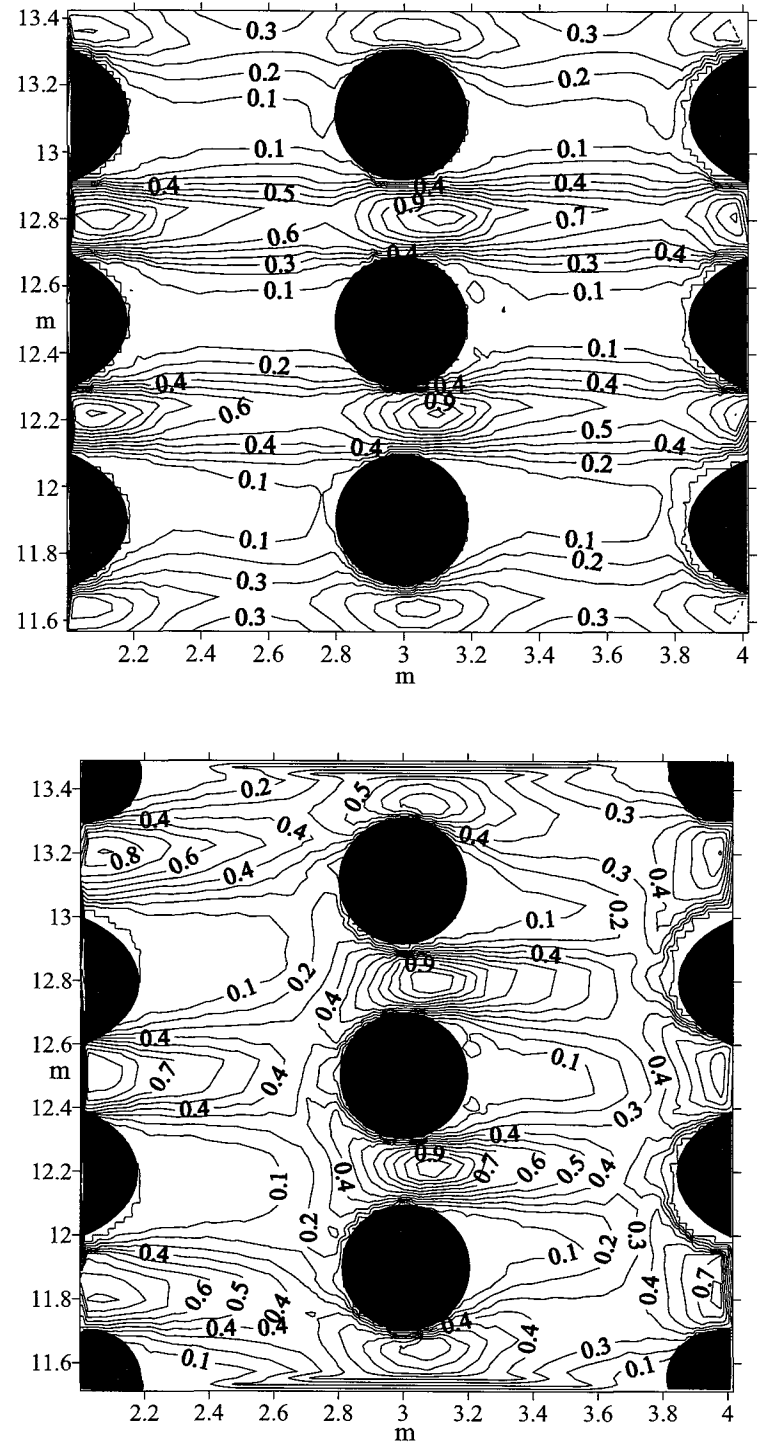

Figure 9 : Equi-velocity lines in fishways embedded with circular obstructions

depth averaged shallow water equations obtained by ignoring vertical accelerations and hence assuming pressure to be hydrostatic.

The test cases perform for model validation shows that the model is able to simulate the flow around obstructions well. Hence, the numerical model can be used confidently to design nature-like fishways embedded with obstructions for lower slopes (upto 5\%). However, regarding the higher slopes, further study is required to establish the limitations and applicability of the model. An utmost care should be taken while selecting the grid size for the computational purpose. The grid convergence test reveals that coarser grids would under-estimate flow velocity. Hence, finer grids should be selected.

This study focused on finding the changes in key flow characteristics, considering a non-submergence case, in nature-like fishways due to the change in shape and pattern of embedment of obstructions. Shorter fish pathways (con-
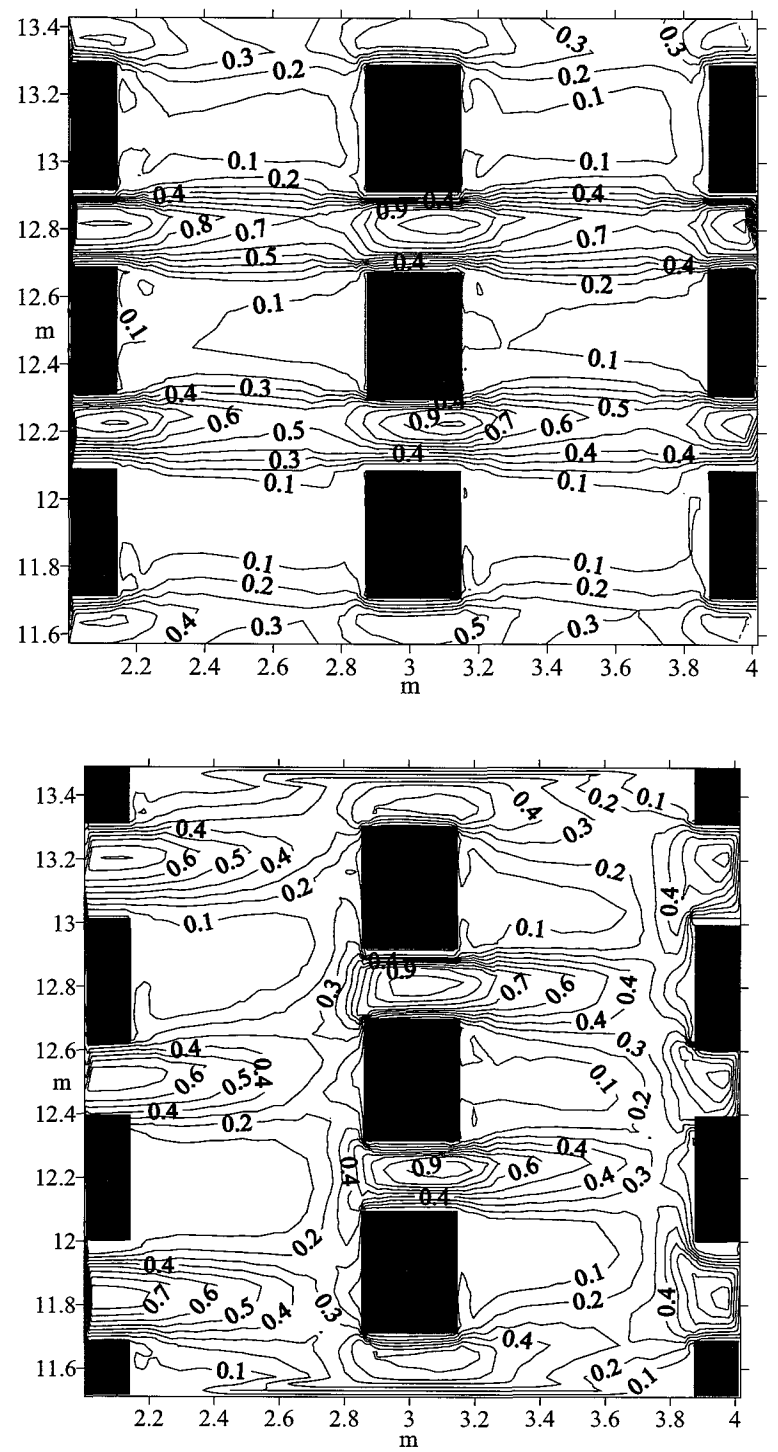

Figure 10 : Equi-velocity lines in fishways embedded with rectangular obstructions

tinuous areas of lower velocities that fish would use to swim upstream or downstream), larger low velocity region (where fish would take rest) and deeper water depths in a pool (for enabling bigger fish to use fishway) are the key factors, among others, that engineers would consider while designing a nature-like fishway.

When the obstructions are embedded with a staggered pattern, flows coming out of the gaps are directly intercepted and diverted by the obstructions ahead. Hence, the maximum velocity is reduced to some extent compared to the regular cases. The diverted flows then mix with the otherwise stagnant flow, behind the obstructions, and reduced the low velocity regions.

When obstructions are embedded with a regular pattern, flows in a fishway exhibited two distinct flow features; flow regions with higher velocities along the gaps and a distinct low velocity pool region behind the obstructions. This pattern of obstructions embedment creates larger pools 

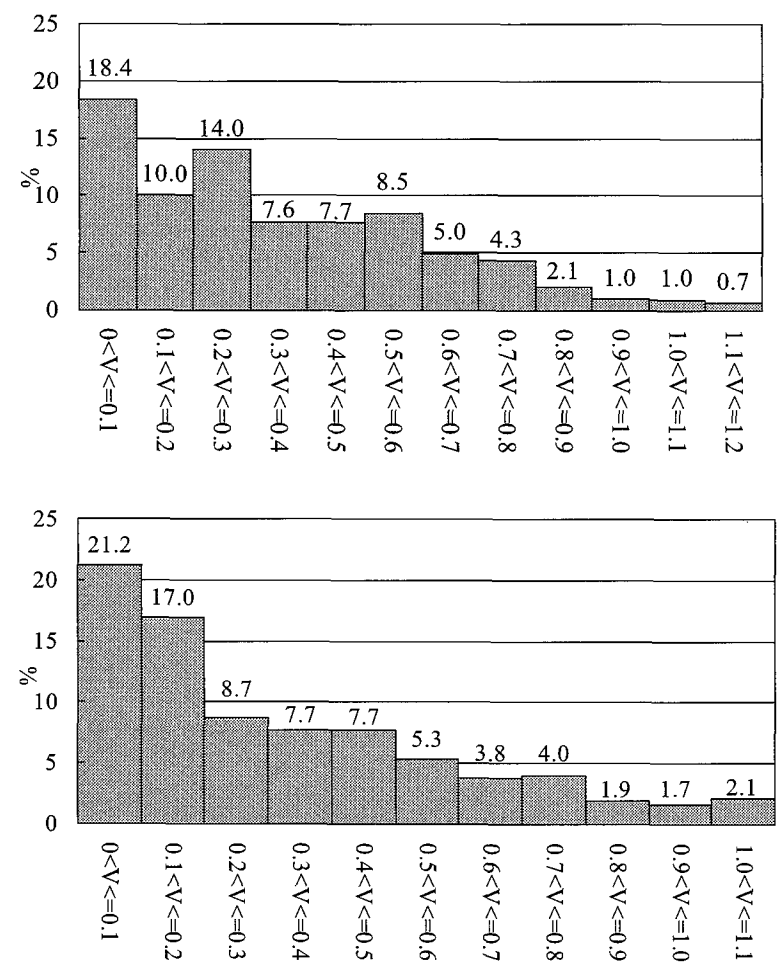

Figure 11 : Velocity distribution in a fishway with circular (top) and rectangular (bottom) obstruction embedded in a regular pattern
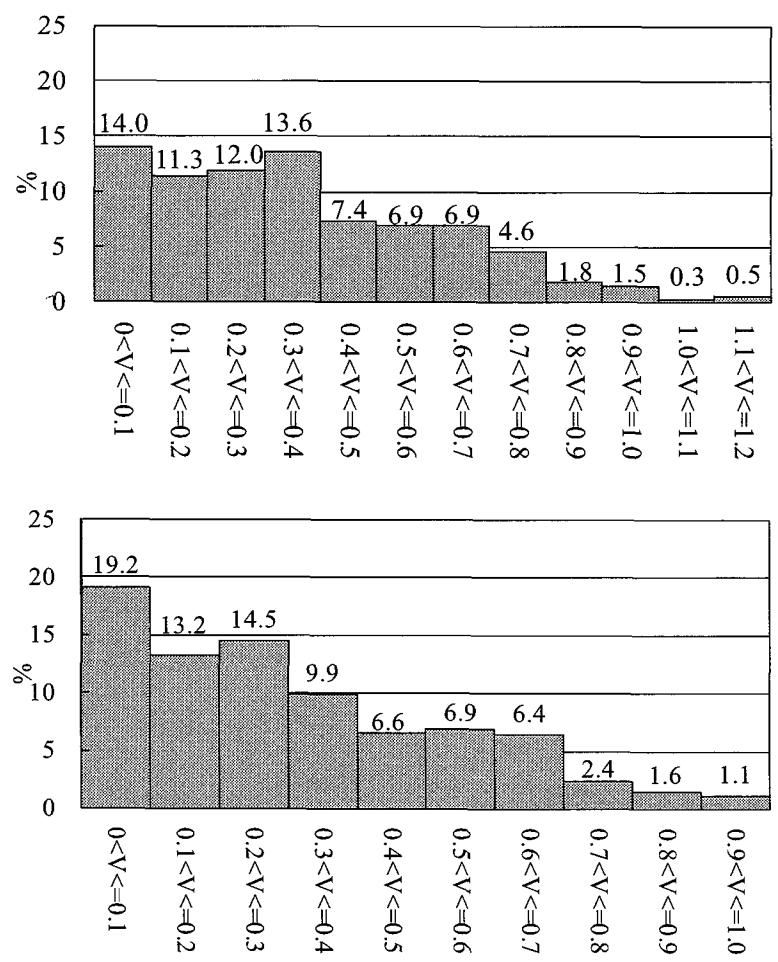

Figure 12 : Velocity distribution in a fishway with circular (top) and rectangular (bottom) obstruction embedded in a staggered pattern

of low velocity regions and a flow region with a little higher velocity at the center of the gaps. Moreover, this pattern
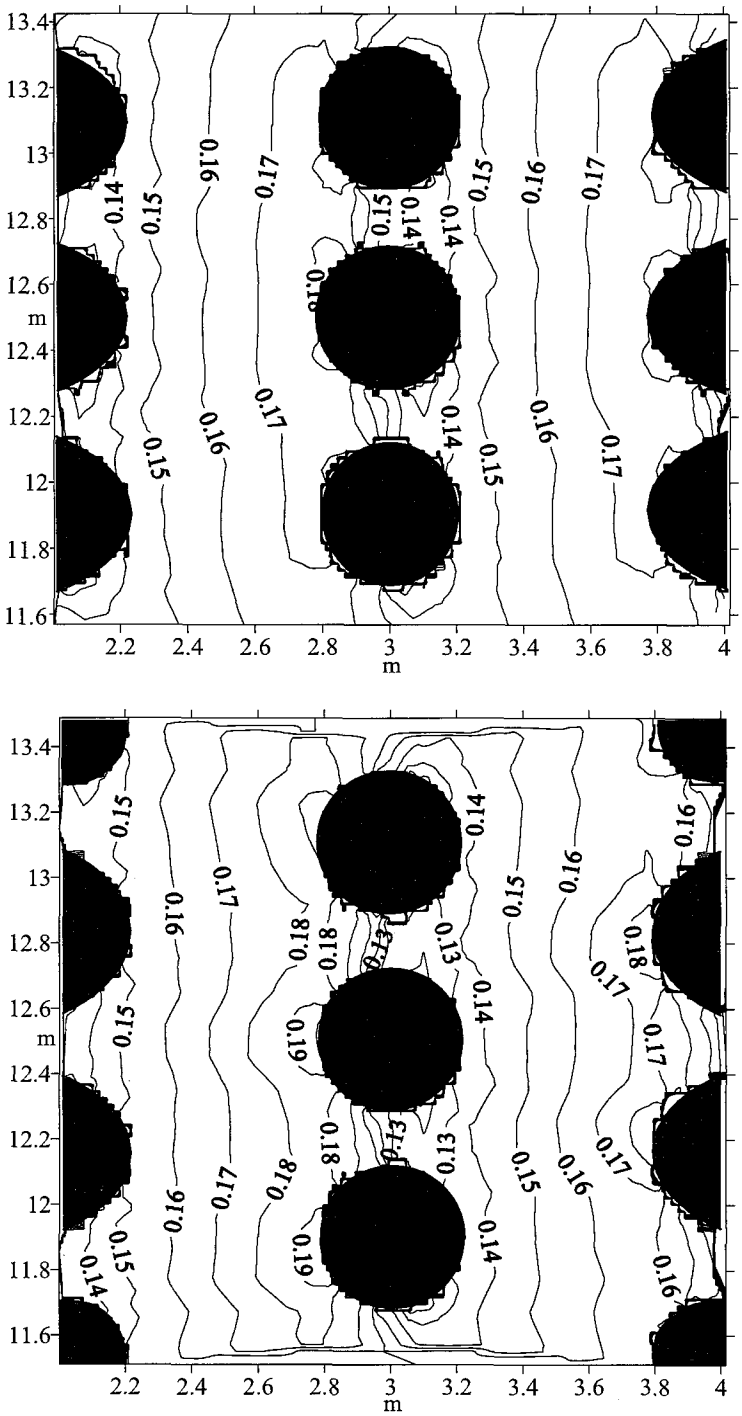

Figure 13 : Water depth contour in fishways embedded with circular obstructions

could provide relatively shorter fish pathways as fish taking refuge in a low velocity region, behind the obstructions, could move to the next low velocity region by swimming along the boundary of the obstructions.

Comparing the circular and rectangular obstructions, higher drag created by rectangular obstructions reduces the maximum velocity by some extent and increases the low velocity region than by circular obstructions. However, the average water depth and water depth distribution patterns, in pools, are almost the same when the obstructions are embedded in a regular pattern.

\section{Conclusions}

This paper presents the application of a Godunov-type numerical method of two dimensional shallow water equations to investigate the flow in nature-like fishways with circular/rectangular obstructions embedded with regular and staggered patterns.

The primary conclusions that could be drawn from the study are as follows:

1. In a nature-like fishway, obstruction embedded with a 

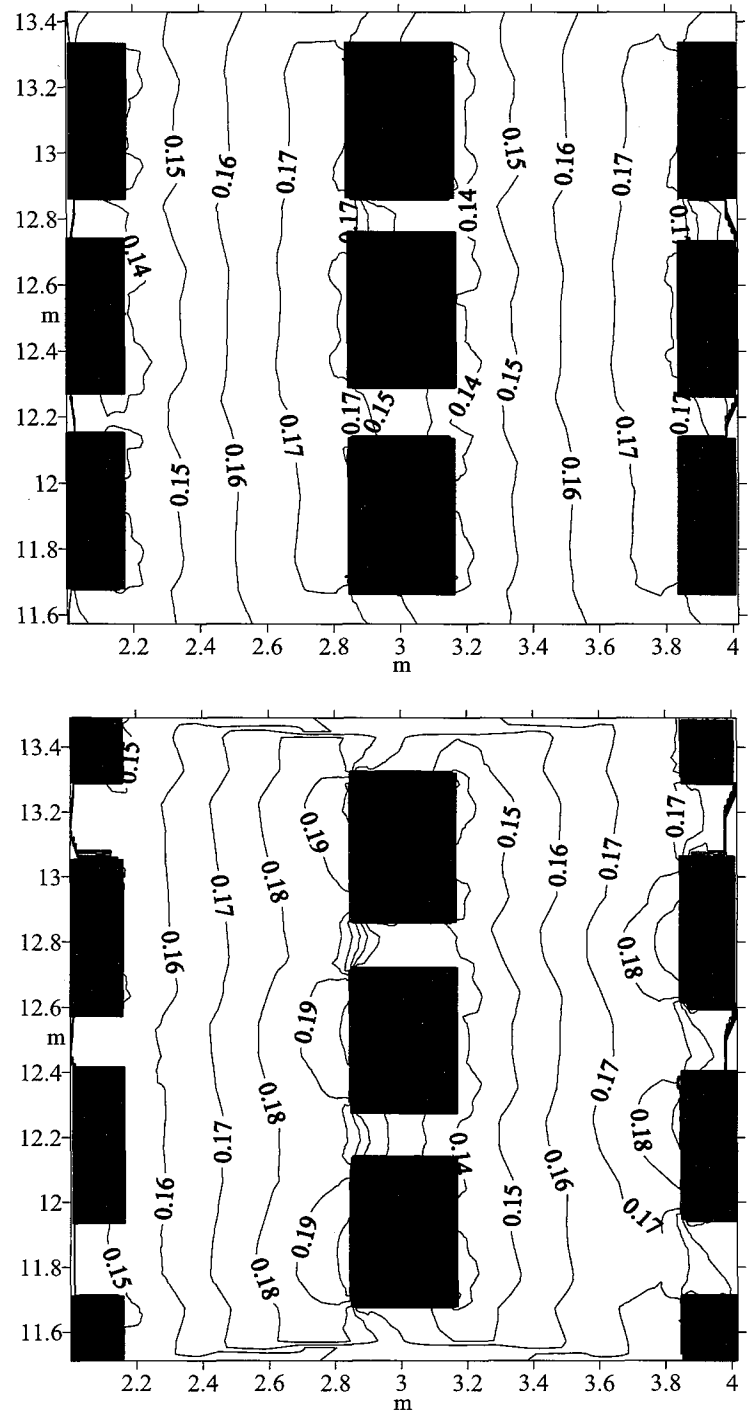

Figure 14 : Water depth contour in fishways embedded with rectangular obstructions

regular pattern provides shorter fish pathways even though the velocity, at the central part of the gap, is a little higher.

2. The obstructions embedded with a regular pattern provide larger low velocity regions, which are indispensable for the design of a better nature-like fishway.

3. Obstructions embedded with a staggered pattern reduce the maximum velocity by some extent. However, the staggered set up of obstructions reduces the low velocity regions and increases the velocities along the upstream perimeter of the obstructions.

4. Maintaining an equal water depth at boundaries, rectangular and circular obstructions, embedded with a regular pattern, creates almost the same average water depth and a very similar depth distribution pattern in a nature-like fishway. However, larger low velocity region is created by the rectangular obstruction when embedded with regular as well as staggered pattern.

\section{Acknowledgements}

The authors would like to thank Dr. J. A. Kells, Department of Civil Engineering, University of Saskatchewan, Canada

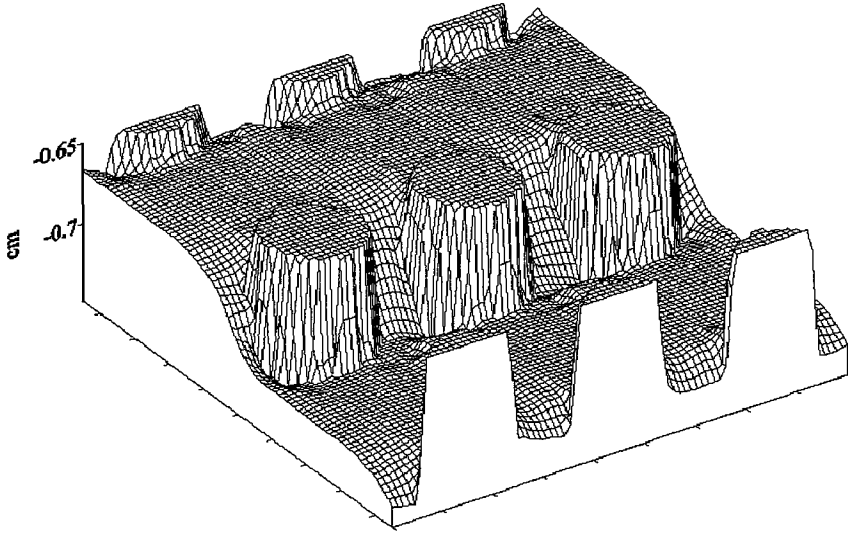

Figure 15 : Free surface configuration in a fishway with circular obstruction embedded in a regular pattern

for providing the literatures of his work related to nature-like fishways.

This work was kindly supported by the Japan Society for the Promotion of Sciences through Grant-in-Aid for Scientific Research No. 18580246. The numerical experiments were conducted by FUJTSU PRIMEPOWER HPC2500 in Institute for Information Management and Communication, Kyoto University.

\section{References}

[1] Acharya, M., Kells, J. A., and Katopodis, C. (2000): Some hydraulic design aspects of nature-like fishways, Proc., Joint Conference on Water resources Engineering and Water resources Planning \& Management, ASCE, Minneapolis, Minnesota, USA., 45.

[2] Akilli, H., Akar, A., and Karakus, C. (2004): Flow characteristics of circular cylinders arranged side-by-side in shallow water, Flow Measurement and Instrumentation., 15(4), pp.187-197.

[3] Bearman, P. W., and Waddock, A. J. (1973): The interaction between a pair of circular cylinders normal to a stream, J. Fluid Mech., 61, pp.499-511.

[4] Harris, J.H., Thornocraft., G., and Werm, P. (1999): Evaluation of rock-ramp fishways in Australia, Proc., Hydraulic engineering for Sustainable water resources management at the Turn of the Millennium, XXVIII IAHR, Vienna, Austria, pp.331-347.

[5] Kells, J. E., Acharya, M., and Katopodis, C. (2000): Design of nature-like fishways for small dam projects." Proc., 3rd Annual Conference, Canadian Dam association, Regina, Canada., pp.85-91.

[6] Kim, H. J., and Durbin, P. A. (1988): Investigation of flow between a pair of circular cylinders in the flopping regime, J. Fluid Mech., 196, pp.431-488.

[7] Puertas, J., Pena, L., and Teijeiro, T. (2004): Experimental approach to the Hydraulics of Vertical Slot Fishways, $J$. Hydraul. Eng., 130(1), pp.10-23.

[8] Rice, C. E., Kadavy, K. C., and Robinson, K. M. (1998): Roughness of loose rock riprap on steep slopes, J. Hydraul. Eng., 124(2), pp.179-185.

[9] Roe, P. L. (1981): Approximate Riemann solvers, parameter vector, and difference schemes, J. Comp. Phys., 43(2), pp.357-372.

[10] Rogers, B., Fujihara, M., and Borthwick, A. G. L. (2001): Adaptive q-tree Godunov-type scheme for shallow water 
equations, Int. J. Numer. Meth. Fluids, 35(3), pp.247-280.

[11] Salaheldin, T. M., Imran, J., and Chaudhry, M. H. (2004): Numerical modeling of the three-dimensional flow field around circular piers, J. Hydraul. Eng., 130(2), pp.91-100.

[12] Schlichting, H. (1979): Boundary-Layer theory, McGraw-Hill, New York, pp.31-32.

[13] Sumner, D., Wong, S. S. T., Price, S. J., and Paidoussis, M. P. (1999): Fluid behavior of side-by-side circular cylinders in steady cross-flow, J. Fluids and Structures, 13(3), pp.309- 338.

[14] Tutar, M., and Holdo, A. E. (2001): Computational modeling of flow around a circular cylinder in sub-critical flow regime with various turbulence models, Int. J. Numer. Meth. Fluids, 35(7), pp.763-784.

[15] Wu, S., Rajaratnam, N., and Katopdis, C. (1999): Structure of flow in vertical slot fishway, J. Hydraul. Eng., 125(4), pp.351-360.

Discussion open until December 31, 2009 